\title{
Avaliação do website móvel e desktop do Sistema Integrado de Bibliotecas da Universidade Federal de Pernambuco
}

\author{
Victor Galvão Celerino Mestre em Ciência da Informação. Universidade Federal de Pernambuco \\ (UFPE) - Brasil. victor.gcx@gmail.com \\ William de Queiroz Tavares Bacharel em Biblioteconomia. Universidade Federal de Pernambuco \\ (UFPE) - Brasil.williamqueiroz7@gmail.com
}

\section{RESUMO}

Este artigo tem como objetivo avaliar as interfaces web (desktop e móvel) do Sistema Integrado de Bibliotecas da Universidade Federal de Pernambuco, com base em duas técnicas de inspeção: Heurísticas de Nielsen e Orientações de Shitkova et al. As Heurísticas de Nielsen serão utilizadas para avaliar a usabilidade do site desktop e as orientações de Shitkova et al. serão aplicadas ao site móvel. A aplicação dessas técnicas apontará os problemas de usabilidade presentes nas interfaces avaliadas e possibilitará uma comparação entre as interfaces. Os resultados obtidos na avaliação do site móvel foram mais satisfatórios do que o site desktop, mesmo este tendo limitações por ser um site móvel uma vez que é oferecido ao usuário diversas outras opções, páginas, links e informações que o auxiliam na navegação e na utilização das ferramentas do site. Ambos os sites se mostraram bem qualificados quanto à questão de usabilidade, necessitando apenas de alguns ajustes que podem ser realizados com a criação de novas funções e o desenvolvimento de documentos.

Palavras-chave: Usabilidade. Usabilidade na web. Heurísticas de Nielsen. Interface móvel.

\section{Evaluation of the mobile and desktop websites of the Integrated System of Libraries of the Federal University of Pernambuco}

\begin{abstract}
This article aims to evaluate the web interfaces (desktop and mobile) Integrated Library System of the Federal University of Pernambuco, based on two inspection techniques: Heuristics Nielsen and Guidelines Shitkova et al. The Nielsen heuristics will be used to evaluate the usability of the desktop site and the guidelines of Shitkova et al. They will be applied to the mobile site. Finally, the application of these techniques will point usability problems present in the evaluated interfaces and enable a comparison between interfaces. The results obtained in the assessment of the mobile site were more satisfactory than the desktop site, even though it has limitations because it is a mobile site since the user is offered several other options, pages, links and information that assist in navigation and in the use of the tools from the website. Both sites proved to be well-qualified on the issue of usability, requiring only a few adjustments that can be made with the creation of new functions and the development of documents.
\end{abstract}

Keywords: Usability. Web Usability. Nielsen Heuristics. Mobile Interface. 


\section{INTRODUÇÃO}

Em meados de 1969, dentro de um cenário militar, a internet começa a surgir com o objetivo de ser usada como uma ferramenta de pesquisa e como um meio de comunicação entre computadores dentro de um ambiente distribuído. Então a Advanced Research Projects Agency Network (ARPANET) foi criada pela Advanced Research Projects Agency (ARPA), um Departamento de Defesa dos Estados Unidos, e tinha como objetivo interligar bases militares e os departamentos de pesquisa em uma rede.

Segundo Eighmey e McCord (1998), a World Wide Web (WWW) é a mídia com o crescimento mais rápido da história. Atualmente, é calculado que na internet existem mais de 1 bilhão de Websites e mais de 3 bilhões de usuários ${ }^{1}$. Com base nesses números, é possível constatar a importância que a internet tem como um canal de comunicação e de informação para o mundo. Segundo Herrera-Viedma et al. (2006), a WWW é atualmente o maior repositório de dados do mundo. E, segundo Porter (2001), a Internet é, em comparação com outras tecnologias, aquela que mais provê oportunidades para as empresas estabelecerem posições estratégicas diferenciadas.

Hoje, quase meio século depois, vemos uma popularização das tecnologias (em particular, de computadores e da internet) e também um aumento e a diversificação dos usuários que as utilizam. Paralelamente, temos o desenvolvimento de novas tecnologias para atender essa diversificação. Diversos sistemas são criados para oferecer aos usuários um nível maior de interatividade, praticidade e rapidez, características presentes na usabilidade.

Usabilidade é definida como a capacidade de um produto ser utilizado por usuários, de maneira eficaz, eficiente e satisfatória, para atingir objetivos específicos (ABNT, 2002). Segundo Cybis, Betiol, Faust (2010), a eficácia se dá a partir do sucesso do usuário para atingir o objetivo; a eficiência está relacionada ao tempo para realização das tarefas; e a satisfação depende do grau de aceitação do sistema pelo usuário.

O conceito de usabilidade trata do estudo de interação entre homem e computador, e é utilizado para qualificar sistemas, sites, ferramentas, aplicações, etc.

Os objetos classificados com alto nível de usabilidade possibilitam o desenvolvimento de tarefas com maior praticidade e velocidade, diminuem o número de erros, aumentam o nível de aprendizado e a satisfação do usuário.

Segundo Bonifácio et al. (2012), dentre as diversas heurísticas utilizadas para a avaliação de sistemas e interfaces, a mais utilizada são as heurísticas propostas por Nielsen

Trata-se de um conjunto de diretrizes aplicadas para a avaliação da usabilidade de qualquer interface.

A metodologia utilizada para a realização da pesquisa será a de estudo de caso, com a avaliação baseada nas Heurísticas de Nielsen. O objetivo geral da pesquisa é avaliar a usabilidade da interface do website móvel $^{2}$ e desktop ${ }^{3}$ do Sistema Integrado de Bibliotecas (SIB), da Universidade Federal de Pernambuco (UFPE), e o objetivo específico é afirmar qual das interfaces mais se adequa as diretrizes de usabilidade.

\section{USABILIDADE}

Com o desenvolvimento tecnológico e a popularização da internet, o número de páginas web aumentou substancialmente e é comum encontrar sites que não possuem uma usabilidade de qualidade. Devido

\footnotetext{
${ }^{1}$ Dados retirados da Internet Live Stats (Disponível em <http://www.internetlivestats.com/>. Acesso em: 30 maio 2016).

${ }^{2}$ http://www.biblioteca.ufpe.br/pergamum/mobile/index.php

3 http://www.biblioteca.ufpe.br/pergamum/biblioteca/index.p
} 
a isso, pesquisadores estudaram e desenvolveram técnicas de inspeção e de observação que possibilitam a melhora da usabilidade em sistemas e interfaces web.

Na literatura sobre Interação Homem-Computador (IHC), usabilidade é a ideia central (LECEROF; PATERNO, 1998; AGARWAL; VENKATESH, 2002). Trata-se da interação entre usuários, programas e aplicações. Mas essa interação não é dada naturalmente, ela é desenvolvida. Para isso, deve-se considerar um conjunto de fatores como: sua interface, suas funcionalidades e seus usuários em potencial. $\mathrm{O}$ que define a usabilidade é a harmonia entre interface, usuário, tarefa e ambiente (CYBIS; BETIOL; FAUST, 2010).

A primeira definição de usabilidade foi apresentada, na norma ISO/IEC 9126 (1991), como "um conjunto de atributos de software relacionados ao esforço necessário para seu uso e para o julgamento individual de tal uso por determinado conjunto de usuários" (DIAS, 2003, p. 3).

Segundo Leite (1998), usabilidade está relacionada à qualidade da interação de sistemas com usuários e depende de diversos aspectos.

A avaliação de usabilidade pode ser realizada a partir de técnicas com o objetivo de maximizar a identificação de problemas de usabilidade. As técnicas são separadas em dois tipos: técnicas de inspeção e técnicas de observação (BARBOSA; SILVA, 2010).

A avaliação de usabilidade pode ser realizada a partir de técnicas com o objetivo de maximizar a identificação de problemas de usabilidade. As técnicas são separadas em dois tipos: técnicas de inspeção e técnicas de observação (BARBOSA; SILVA, 2010).

Nas técnicas de inspeção, o avaliador investiga e identifica problemas de usabilidade presentes nas interfaces dos sistemas, sem o envolvimento direto do usuário. As técnicas se baseiam em checklists que são utilizados em especificações de interfaces, protótipos ou sistemas completos. Os exemplos existentes que utilizam essas técnicas são: a Avaliação Heurística, o Percurso Cognitivo e a Inspeção Semiótica

As técnicas de observação permitem identificar os problemas reais dos sistemas, porque a avaliação é realizada no momento de interação entre usuário e sistema. Como exemplos de técnicas de observação temos: os Testes de Usabilidade, as Avaliações de Comunicabilidade e a Prototipagem.

Segundo Shneiderman (1998), usabilidade é a propriedade de uma interface que permite o usuário classificá-la quanto aos aspectos de qualidade. Essa classificação se dá a partir de cinco atributos:

- Facilidade de aprendizado: o usuário consegue realizar tarefas em prazos curtos;

- Eficiência de uso/desempenho na execução de tarefas: o usuário, quando domina o sistema, consegue realizar as tarefas com alto grau de produtividade;

- Retenção com o tempo: trata-se da adaptação do usuário com o sistema, isso deve levar em consideração até usuários menos experientes;

- Minimização de erros: está relacionada à taxa de erros durante a utilização. É importante que o sistema consiga reverter os erros cometidos pelo usuário;

- Satisfação: o sistema tem que ser agradável de se usar.

\subsection{USABILIDADE NA WEB}

Com o crescente número de páginas da web é comum encontrarmos problemas relacionados à usabilidade, logo, é necessário que as páginas da web corrijam esses problemas para proporcionar aos usuários facilidade e praticidade.

Nielsen e Loranger (2007) apontam algumas diretrizes que devem ser observadas quanto à usabilidade de uma página web. Dentre essas diretrizes é importante destacar oito que, mesmo apontadas em pesquisas conduzidas em 1994, mostram-se bastante atuais.

1. Mudança de cor dos links visitados: é importante que durante a navegação na página web o usuário consiga identificar por onde ele já passou, onde está e onde pode ir. Páginas que não alteram a cor do link após acessado causam confusão e deixam o usuário perdido. Esse problema é ainda mais grave para os usuários com dificuldade em lembrar o caminho realizado para chegar em determinada página (memória de curto prazo). 
2. Botão voltar: a possibilidade de voltar à página anterior após acessar outra é uma das características essenciais em páginas web. Mesmo com tamanha importância dessa função, alguns sites não disponibilizam o botão voltar para o usuário e isso dificulta a navegação, deixando o usuário "preso" ou até mesmo causando a desistência da utilização da página.

3. Abertura de links em novas janelas: em alguns sites, ao clicar em um link ele é aberto em uma nova janela, e isso pode ser uma complicação, pois vários usuários encontram dificuldades em manipular diversas janelas que foram abertas de forma indesejada. É recomendado que, por padrão, os links abram na mesma janela e que exista o botão voltar.

4. Pop-up: é um dos maiores causadores de irritação nos usuários. As janelas pop-up que aparecem, frequentemente de surpresa, atrapalham a navegação do usuário. Em sua maioria, mudam o foco do usuário para informações que não são de seu interesse (anúncios).

5. Anúncios e Publicidade: é comum que usuários ignorem os anúncios e identifiquem apenas o que é importante. Para os usuários, a informação, em sua maioria, se apresenta em textos simples, e quando anúncios se apresentam da mesma forma isso lhes causa irritação.

6. Padrões Web: os usuários se acostumam com certos padrões de interface de sites e esperam encontrar esse mesmo padrão em outros sites. Quando isso não ocorre, é comum que os usuários se sintam confusos e tenham que se acostumar a uma nova interface.

7. Conteúdo vago: é importante apresentar o conteúdo de forma simples e objetiva, evitando textos rebuscados e sem valor, pois alguns usuários descartam esse tipo de material.

8. Conteúdo denso: $O$ texto presente nos sites deve ser breve, selecionável e acessível, pois isso evita o cansaço e a desistência por parte do usuário.

Nielsen e Loranger (2007) apontam, também, alguns aspectos relacionados ao design e ao conteúdo que são determinantes para uma boa navegabilidade nas páginas da web. Tais aspectos são:

- Elementos comuns de Design: tem a intenção de informar ao usuário onde ele está localizado e onde pode ir. É indicada a inclusão de três elementos em cada página do website: nome da instituição, localizado no canto superior esquerdo; link de acesso direto para a página principal; e campo de busca no canto superior direito.

- Homepage: é a página principal do website e deve informar ao usuário onde ele está, onde pode ir e as opções de todas as seções do site.

- Buscas: um sistema de buscas é essencial, pois através dele o usuário pode refinar melhor o que procura, proporcionando um acesso mais amplo e fácil ao conteúdo.

- Rolagem: é recomendado não deixar a rolagem das páginas muito extensa. É comum os usuários perderem o interesse no site quando ele apresenta mais de 2,5 telas de rolagem.

- Textos: recomenda-se estudar, antes, quais os tipos de fonte e tamanho dos textos agradam mais o usuário do site, pois não existe um padrão que agrade a todos. Nielsen e Loranger (2007) recomendam que o tamanho de texto seja entre 10 e 12, e a utilização da fonte sem serifa porque são mais rápidas de ler em telas de computador

\section{HEURÍSTICAS PARA AVALIAÇÃO DE USABILIDADE}

Infelizmente é comum em muitos sites a presença de problemas de design que dificultam a usabilidade. Segundo Santa Rosa e Moraes (2008), esses problemas devem-se ao fato de que muitos desenvolvedores web foram treinados apenas para a utilização das ferramentas para a autoria de websites.

Para evitar esses problemas é indicada a realização de avaliações das interfaces. A avaliação heurística é composta por uma lista de princípios utilizados para investigar e identificar problemas de usabilidade na interface e têm a vantagem de poder ser realizada a qualquer momento, seja na concepção do sistema ou após a sua criação (ANDRADE, 2007).

Em sua maioria, as avaliações são realizadas por um avaliador, mas é recomendado até três, pois é possível que apenas um não consiga identificar todos os problemas de usabilidade presentes na interface. 
Segundo Nielsen e Loranger (2007), quando o número de avaliadores é muito grande, é comum a indicação dos mesmos problemas de usabilidade, ou seja, não é necessária a presença de muitos avaliadores.

Atualmente existem diversas diretrizes e princípios propostos por diversos pesquisadores para a avaliação de usabilidade de interfaces, mas os que serão utilizados para o desenvolvimento da avaliação dos websites serão as heurísticas propostas por Nielsen e as orientações apresentadas por Shitkova et al. (2015).

\subsection{HEURÍSTICAS DE NIELSEN}

A avaliação heurística de Nielsen foi criada em 1994 em conjunto com Rolf Molich. Nielsen e Molich (CRUZ; SOARES NETO, 2015). Definem as heurísticas como um método de avaliação de usabilidade utilizado para a identificação de problemas de usabilidade presentes em interfaces.

Segundo Nielsen e Loranger (2007), com base na avaliação de usabilidade proposta pela sua heurística, é possível afirmar se a interface apresenta uma boa usabilidade. Os 10 princípios propostos por Nielsen para avaliação de usabilidade de interface são:

1. Visibilidade do estado do sistema: manter o usuário sempre informado sobre o que está acontecendo. A interface informa onde o usuário está, de onde veio e para onde vai. É importante que exista um feedback.

2. Compatibilidade entre o sistema e o mundo real: utilização da linguagem do usuário ao invés da linguagem do sistema. As instruções devem ser indicadas de forma lógica e natural para que façam sentido ao usuário.

3. Liberdade e controle ao usuário: trata-se da possibilidade de fornecer ao usuário uma "saída de emergência" para situações onde são ativadas funções do sistema por engano. Ou seja, suporte às funções como: fazer e desfazer.

4. Consistência e padrões: evitar a utilização de palavras ou ações diferentes que signifiquem a mesma coisa. A interface deve utilizar de padrões para a utilização de palavras, cores, posições de itens, para obter uma coerência em todas as telas do sistema.

5. Prevenção de erros: evitar a ocorrência de erros, pois são fontes de frustração, ineficiência e ineficácia do sistema.

6. Reconhecimento em vez de relembrar: tornar objetos, ações e opções visíveis e coerentes. As instruções de uso devem ser visíveis e de fácil acesso.

7. Flexibilidade e eficiência de uso: o sistema deve oferecer aos usuários, experientes ou não, opções que auxiliem nas suas tarefas, como atalhos de teclas, opções avançadas de busca, filtro, etc.

8. Projeto minimalista e estético: evitar o uso de informações desnecessárias e disponibilizá-las de forma natural e agrupadas quando tiverem relação, permitindo uma melhor visibilidade do sistema.

9. Auxiliar os usuários a reconhecer, diagnosticar e recuperar erros: apresentar mensagens de erro em linguagem natural do usuário (evitar uso de códigos), indicando possíveis soluções para o problema.

10. Ajuda e documentação: oferecer ao usuário informações que auxiliem na utilização do sistema. Essa ajuda deve ser facilmente localizada, de fácil compreensão, estruturada e não ser muito complexa.

\subsection{ORIENTAÇÕES PARA AVALIAÇÃO DE SITES MÓVEIS}

A avaliação de sites móveis e sites desktop deve ser feita de forma diferente, já que os ambientes nos quais eles se apresentam possuem limitações diferentes umas das outras, como: resolução, velocidade, ferramentas, estrutura, navegação, design, conteúdo, etc. 
Em um estudo realizado pelos pesquisadores Shitkova et al. (2015), foram apresentadas orientações que possibilitam a avaliação da usabilidade de sites móveis. Essas orientações abordam os seguintes pontos:

Quadro 1 - Orientações para usabilidade de sites móveis

\begin{tabular}{|l|}
\hline \multicolumn{1}{|c|}{ Layout } \\
\hline$\left(\mathrm{O}_{1}\right)$ - Conteúdo colocado na parte central da tela. \\
$\left(\mathrm{O}_{2}\right)$ - Evita a rolagem horizontal. \\
$\left(\mathrm{O}_{3}\right)$ - Organizar o conteúdo verticalmente, evitar o uso de tabs. \\
$\left(\mathrm{O}_{4}\right)$ - Botão login localizado no topo da tela. \\
$\left(\mathrm{O}_{5}\right)$ - Campo de busca visível e usável. \\
$\left(\mathrm{O}_{6}\right)$ - Usar uma estrutura linear clara e evita a estrutura da tabela de campos de entrada. \\
$\left(\mathrm{O}_{7}\right)$ - Não use mais de três botões em um diálogo. \\
$(\mathrm{O} 8)$ - Posição botões no meio ou no fim do diálogo. \\
$\left(\mathrm{O}_{9}\right)$ - Posição dos botões no lado direito da tela.
\end{tabular}

(O10) - Menu de navegações fáceis e simples.

Navegação

(O11) - Menus de navegação de nível um.

(O12) - Evitar a utilização de muitas opções. Utilizar no máximo 4-8 itens e de rolagem no menu de navegação.

(O13) - Páginas importantes acessíveis a partir da página inicial.

(O14) - Possibilidade de retornar rapidamente para a página inicial.

(O15) - Minimizar o número de cliques necessários para alcançar cada página.

(O16) - Apresenta os caminhos percorridos pelo usuário e possibilita o usuário retornar rapidamente à página inicial e alternar entre as páginas.

(O17) - Abre links externos em uma nova janela, mantendo a janela atual inalterada.

(O18) - Menu estruturado por tópicos.

(O19) - Títulos, links e itens de navegação não ambíguos, autoexplicativos e bem descritos.

\section{Design}

(O20) - Design simples, coerente, uniforme e claro.

(O20) - Design simples, coerente, uniforme e claro.

(O21) - Utilizar mesmo modelo para diferentes representações do mesmo produto (por exemplo, website e app 4 móvel).

(O22) - Destaca informações importantes.

$\left(\mathrm{O}_{23}\right)$ - Uso de ícones fáceis de identificar e visíveis.

$\left(\mathrm{O}_{24}\right)$ - Botões entre 7 e 10 milímetros.

\section{Conteúdo}

(O25) - Conteúdo e funcionalidade semelhantes tanto para a versão móvel como para a versão desktop.

(O26) - Evitar textos longos e usar frases simples.

(O27) - Não mostrar muita informação na página inicial.

(O28) - Conteúdo ordenado por sua importância.

(O29) - Fornece sugestões automáticas dentro do aplicativo (por exemplo, preenchimento automático de busca).

(O30) - Permite que as ações do usuário sejam revertidas para realização de correções rápidas.

(O31) - Apresenta diálogos de confirmação para ações de mudança e edição.

(O32) - Evita a perda de dados, lembrando os usuários de alterações não salvas.

(O33) - O estado atual do aplicativo é mantido mesmo quando minimizado e restaurado novamente.

(O34) - Correção automática dos erros de digitação.

${ }_{4}^{4}$ Abreviação em inglês para a palavra applications; Tradução: aplicativos. 
$\left(\mathrm{O}_{35}\right)$ - Interface otimizada para evitar o toque impreciso.

$\left(\mathrm{O}_{3} 6\right)$ - Apresenta feedback tácteis sobre as ações do usuário.

$\left(\mathrm{O}_{37}\right)$ - Suporte a gestos comuns de controle das aplicações.

$\left(\mathrm{O}_{3} 8\right)$ - Entrada fácil de senhas em dispositivos móveis.

(O39) - Tempo de carregamento curto.

Performance

Fonte: Shitkova et al. (2015).

As orientações propostas por Shitkova et al. (2015) possuem algumas similaridades com as heurísticas de Nielsen. Além dessas semelhanças, são apresentados alguns aspectos que só podem ser aplicados a sites móveis, como os feedback tácteis, tamanho e localização dos botões, etc.

\section{METODOLOGIA}

O Sistema Integrado de Bibliotecas da Universidade Federal de Pernambuco (SIB UFPE) é composto pelas 14 bibliotecas localizadas nos centros acadêmicos da UFPE. Juntas, possuem em sua coleção mais de 300 mil títulos e mais de 1 milhão de exemplares. Os seus objetivos são difundir informação, democratizar o conhecimento acadêmico e apoiar as atividades de ensino, pesquisa e extensão da UFPE.

O SIB UFPE utiliza um software de gerenciamento de bibliotecas chamado Pergamum. Esse software foi criado em 1988 a partir de um Trabalho de Conclusão de Curso de Ciência da Computação do Centro de Ciências Exatas e Tecnológicas da Pontifícia Universidade Católica do Paraná (UFCPR), com o objetivo de possibilitar que cada instituição seja capaz de gerenciar qualquer tipo de documento e atender diversas unidades informacionais, sejam elas bibliotecas universitárias, escolares, institucionais, etc.

Atualmente, o Pergamum oferece para as unidades informacionais a possibilidade de apresentar o seu site em dois formatos: desktop e móvel. O trabalho pretende avaliar a usabilidade dos dois formatos apresentados pelo SIB UFPE, com o objetivo de mostrar se atendem, de forma adequada, as heurísticas e orientações propostas na literatura sobre a usabilidade de websites.

Trata-se de um trabalho qualiquantitativo que tem como metodologia o estudo de caso, pois pretende fazer uma avaliação com base nas heurísticas de Nielsen e nas orientações de Shitkova et al. (2015). As páginas que serão avaliadas no site desktop e móvel serão: a página inicial, a página de busca e a página de login.

O site desktop será avaliado com base nas heurísticas de Nielsen e a avaliação será feita através da atribuição de notas baseadas no grau de severidade do problema (Quadro 2).

Quadro 2-Grau de severidade

\begin{tabular}{|c|l|l|}
\hline $\begin{array}{c}\text { Grau de } \\
\text { severidade }\end{array}$ & \multicolumn{1}{|c|}{ Tipo } & \multicolumn{1}{c}{ Descrição } \\
\hline 0 & Sem importância & Não afeta a operação da interface \\
\hline 1 & Cosmético & Não há necessidade imediata de solução \\
\hline 2 & Simples & Problema de baixa prioridade (pode ser reparado) \\
\hline 3 & Grave & Problema de alta prioridade (deve ser reparado) \\
\hline 4 & Catastrófico & Muito grave, deve ser reparado de qualquer forma. \\
\hline
\end{tabular}

Fonte: Nielsen (2007)

O site móvel, por se apresentar em uma plataforma diferente, será avaliado através das orientações propostas por Shitkova et al. (2015). A avaliação irá apresentar se o site móvel segue algumas dessas orientações, indicando se foram ou não obedecidas.

\section{AVALIAÇÃO DO SITE DESKTOP A PARTIR DAS HEURÍSTICAS DE NIELSEN)}


A avaliação do site desktop foi estruturada com base nas heurísticas de Nielsen. Os resultados das avaliações das três páginas selecionadas serão apresentados, heurística por heurística. Portanto:

1. Visibilidade do estado do sistema - O grau de severidade atribuído nessa categoria é o. Nas três páginas, a localização de onde o usuário está é bem visível e o sistema apresenta um feedback em tempo real do que está acontecendo para o usuário.

2. Compatibilidade entre o sistema e o mundo real - Em todas as páginas o sistema apresenta uma linguagem padronizada e coerente, o que proporciona mais qualidade à navegação. $\mathrm{O}$ grau de severidade foi o.

3. Liberdade e controle ao usuário.

4. O grau de severidade atribuído é o, já que, em todas as páginas, o sistema permite que o usuário cancele ou refaça as suas ações. Por exemplo: é possível cancelar a realização de uma busca, a escolha de filtros, etc.

5. Consistência e padrões.

6. Nas páginas avaliadas, o sistema se apresenta bem consistente, pois não existem botões ou textos que têm a mesma função. O grau de severidade desse problema é o.

7. Prevenção de erros.

8. Durante a navegação, não houve, em nenhum momento, a oferta de algum sistema ou ferramenta que prevenisse os possíveis erros praticados pelo usuário ou ocasionados pelo próprio sistema. Portanto, devido a importância desse item, o grau de severidade atribuído é 2.

9. Reconhecimento em vez de relembrar.

10. O site possui um público específico que, normalmente, no primeiro ou segundo acesso, consegue lembrar das funções oferecidas pelo sistema sem ter que procurar novamente pelo site. Mas, infelizmente, a localização de algumas opções, não está bem destacada para o usuário, fazendo com que ele tenha que relembrar e procurar novamente por essa opção. Portanto, o grau de severidade do problema é 1.

11. Flexibilidade e eficiência de uso.

12. O sistema oferece, de forma simples e visível, opções que auxiliam na flexibilidade e eficiência de uso por parte do usuário, como: pesquisa avançada, filtros, classificação, etc. O grau de severidade é o.

13. Projeto minimalista e estético.

Em todas as páginas é respeitado um design minimalista. Os textos utilizados são bem simples, mas indicam de forma direta do que se trata. Porém, na página inicial, existe uma certa "poluição" visual, com a presença de imagens e textos que poderiam ser deslocados para outra página (Figura 1). O grau de severidade é 1.

Figura 1 - Poluição 


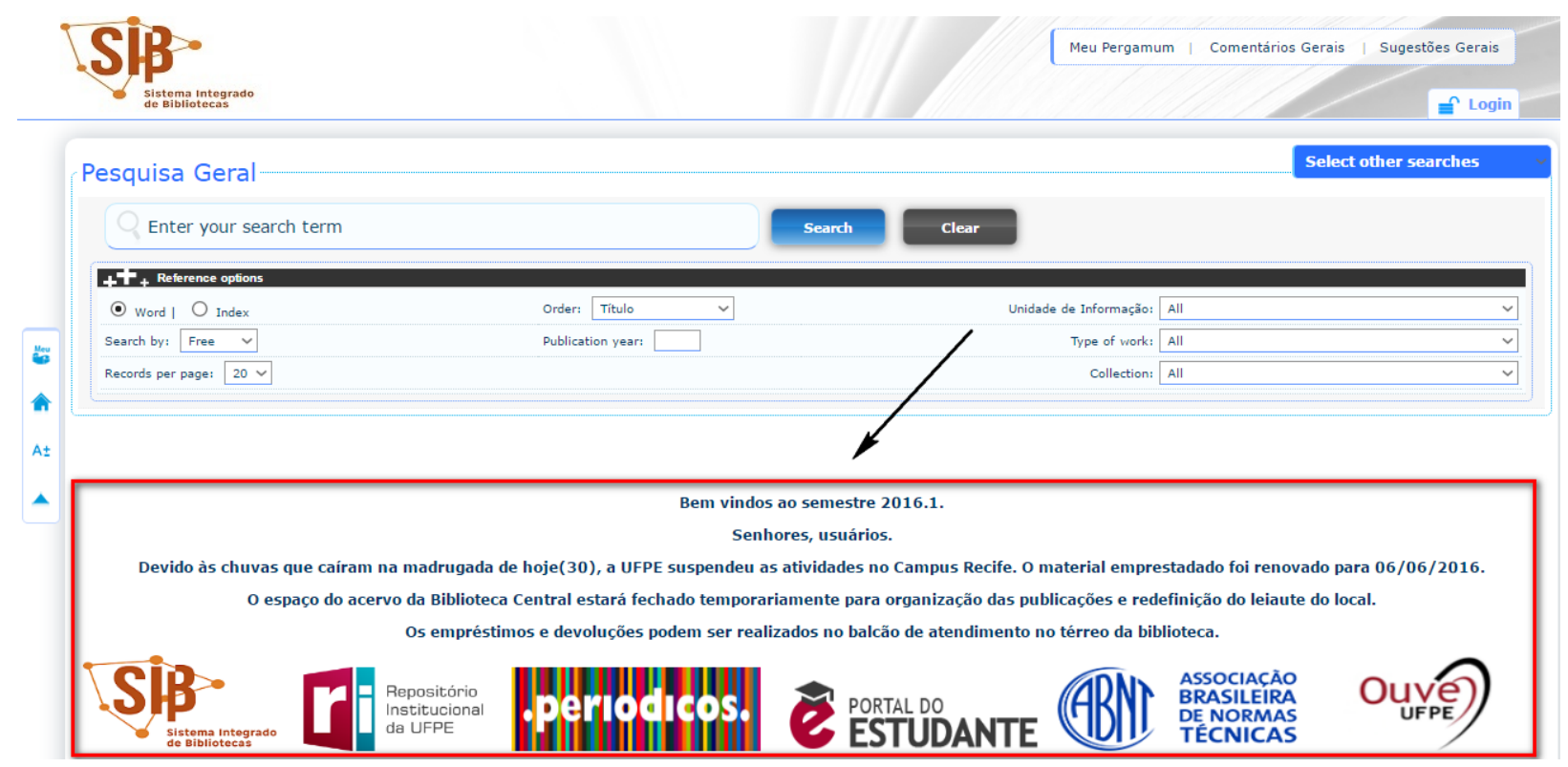

Fonte: Autoria nossa (2018).

1. Auxiliar os usuários a reconhecer, diagnosticar e recuperar erros.

2. O sistema oferece apenas a opção de o usuário interromper as suas ações, mas não apresenta formas do usuário se recuperar desses erros. O grau de severidade é 2, pois, assim como a prevenção de erros, essa heurística é muito importante de ser seguida, já que permite ao usuário se recuperar dos erros cometidos ao utilizar o sistema.

3. Ajuda e documentação.

4. É de extrema importância que o site ofereça ajuda ao usuário através de documentos ou textos inseridos na página. Infelizmente, em todas as páginas, o sistema não ofereceu, em nenhum momento, textos ou documentos que ajudassem o usuário quanto à utilização do sistema ou quanto à reparação de erros. Devido à importância dessa característica, o grau de severidade é 3 .

Conforme explicado na metodologia, a avaliação do site móvel foi feita com base nas orientações de Shitkova et al (2015). Para a realização dessa avaliação, foi criado um quadro onde serão indicadas cada uma das orientações apresentadas no capítulo 3.2, e serão atribuídos indicadores, como: OK (o sistema segue a orientação), X (o sistema não segue a orientação) e N (orientação não se aplica).

É possível observar, na figura 2, em ordem vertical, a página inicial, a página de login e a página de busca do site móvel. A avaliação foi aplicada nas três páginas que, de forma geral, se saíram bem (quadro 3 ). Entretanto, é importante destacar alguns problemas apresentados em sua interface.

Figura 2 - Página inicial, login e busca 


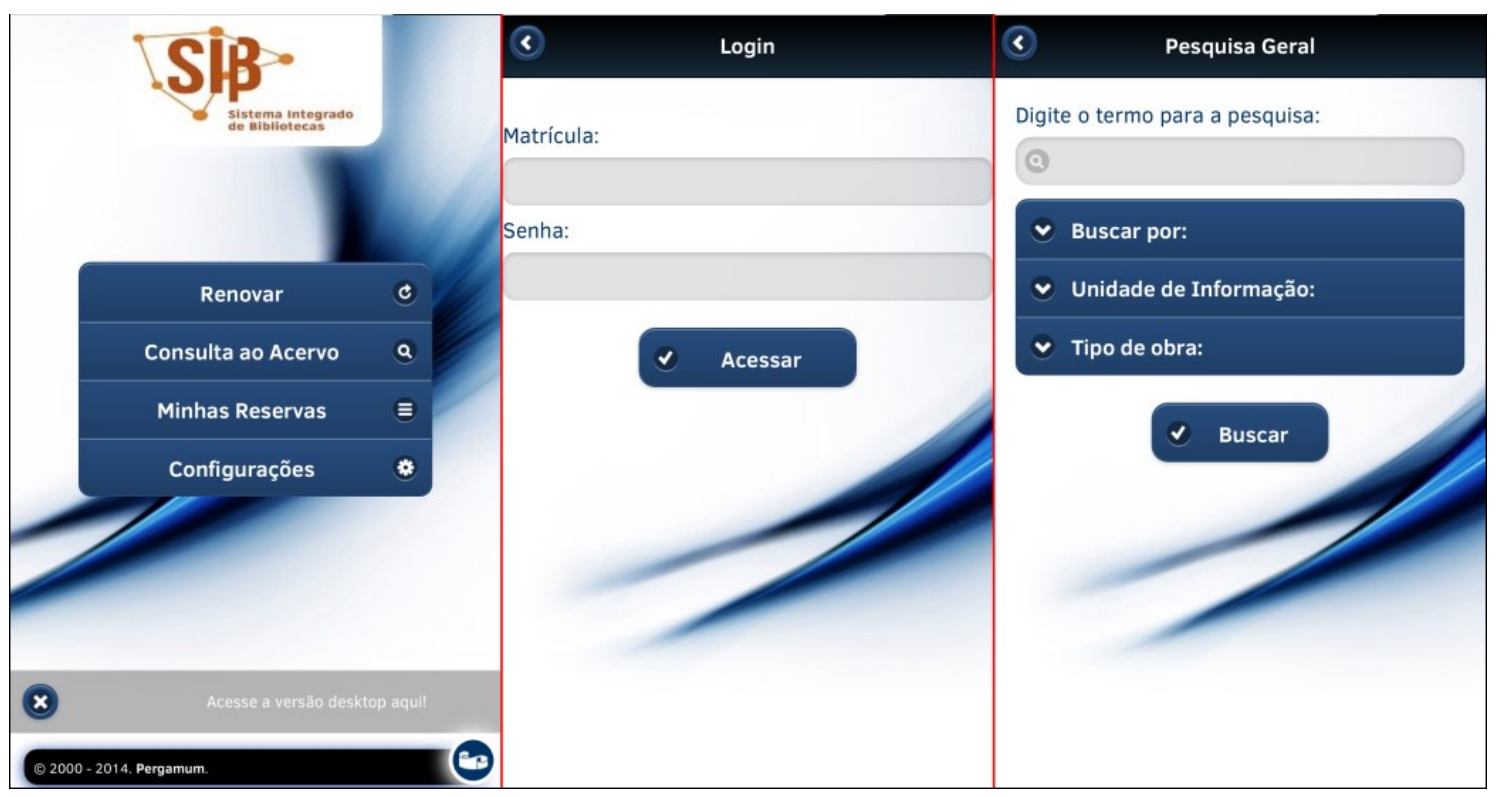

Fonte: Autoria nossa (2018)

Quadro 3-Avaliação

\begin{tabular}{|c|c|c|c|c|c|c|c|c|c|c|}
\hline Layout & $\mathrm{O}_{1}$ & $\mathrm{O}_{2}$ & $\mathrm{O}_{3}$ & $\mathrm{O}_{4}$ & $\mathrm{O}_{5}$ & 06 & $\mathrm{O}_{7}$ & 08 & \multicolumn{2}{|c|}{ O9 } \\
\hline Resultado & OK & OK & OK & OK & OK & OK & OK & OK & \multicolumn{2}{|c|}{$\mathrm{N}$} \\
\hline Navegação & O10 & O11 & O12 & $\mathrm{O}_{13}$ & $\mathrm{O}_{14}$ & O15 & 016 & O17 & 018 & O19 \\
\hline Resultado & OK & OK & OK & OK & OK & OK & $x$ & $X$ & OK & OK \\
\hline Design & $\mathrm{O}_{20}$ & $\mathrm{O}_{21}$ & $\mathrm{O} 22$ & $\mathrm{O}_{23}$ & \multicolumn{6}{|c|}{024} \\
\hline Resultado & OK & $\mathrm{N}$ & OK & OK & \multicolumn{6}{|c|}{ OK } \\
\hline Conteúdo & $\mathrm{O}_{25}$ & $\mathrm{O} 26$ & $\mathrm{O} 27$ & $\mathrm{O} 28$ & O29 & $\mathrm{O}_{30}$ & $\mathrm{O}_{31}$ & $\mathrm{O}_{32}$ & \multicolumn{2}{|c|}{$\mathrm{O}_{33}$} \\
\hline Resultado & OK & OK & OK & OK & $x$ & $x$ & OK & OK & \multicolumn{2}{|c|}{ OK } \\
\hline Conteúdo & $\mathrm{O}_{34}$ & $\mathrm{O}_{35}$ & $\mathrm{O}_{36}$ & $\mathrm{O}_{37}$ & \multicolumn{6}{|c|}{$\mathrm{O}_{38}$} \\
\hline Resultado & $x$ & OK & $x$ & OK & \multicolumn{6}{|c|}{ OK } \\
\hline Performance & \multicolumn{10}{|c|}{$\mathrm{O}_{39}$} \\
\hline Resultado & \multicolumn{10}{|c|}{ OK } \\
\hline
\end{tabular}

Fonte: Dados da pesquisa (2018)

As orientações que a interface móvel apresentou problema foram as de número: 16, 17, 29, 30, 34 e 36 . Os problemas de número 16 e 17 são de navegação. Em nenhum momento o site móvel apresentou uma orientação dos caminhos percorridos no sistema, causando assim um problema de localização para o usuário. O problema 17 se refere à abertura de links em novas abas. O site em si não oferece essa possiblidade, todos os links são abertos sempre na mesma aba e, como também não apresenta os caminhos percorridos, isso aumenta ainda mais o problema de localização do usuário.

Os problemas 29, 30, 34 e 36 estão relacionados ao conteúdo exibido no site. O site, tanto o móvel como o desktop, não apresentam ao usuário as possibilidades de preenchimento automático (O29) e de correção automática dos erros de digitação $\left(\mathrm{O}_{34}\right)$. Em contrapartida ao site desktop, o site móvel não oferece ao usuário a possiblidade de reverter ou cancelar suas ações ( $\mathrm{O}_{30}$ ), problema grave, já que, sem querer, algumas ações podem ser realizadas. Quanto ao feedback táctil $\left(\mathrm{O}_{3} 6\right)$, o site móvel não dispõe dessa funcionalidade, ou seja, dificulta a usabilidade do site para alguns usuários. 


\section{CONCLUSÃO}

No decorrer do trabalho percebe-se a importância que a usabilidade tem para os sistemas e sites, pois quando seguem orientações ou padrões, com objetivo de melhorar a sua usabilidade, tornam-se mais acessiveis, fáceis de usar e de aprender.

É evidente que o trabalho de melhorar a usabilidade de um sistema é bastante árduo, mas, sem dúvidas, esse trabalho irá agregar mais qualidade ao sistema.

Durante a avaliação do site desktop foram encontrados diversos problemas, mas, em sua maioria, simples e de fácil resolução, exceto o problema de Ajuda e Documentação, que foi classificado em grau 3. Esse problema é de fácil solução, mas é muito grave, pois é muito importante para os usuários, seja ele novo ou experiente.

Os resultados obtidos na avaliação do site móvel foram mais satisfatórios, pois ele possui um grau de usabilidade superior ao do site desktop, mesmo tendo limitações por ser um site móvel. É importante destacar que o problema $\mathrm{O}_{30}$, referente à reversão de erros cometidos pelo usuário, foi o único problema grave encontrado durante a sua avaliação.

Ao comparar o site desktop com o site móvel é evidente que o desktop, mesmo apresentando alguns problemas, é superior ao móvel, uma vez que são oferecidas ao usuário diversas outras opções, páginas, links e informações que o auxiliam na navegação e na utilização das ferramentas do site.

A vantagem do site móvel é que, por possuir um design mais minimalista, ele direciona melhor os usuários que desejam realizar as operações principais do site, como: reservas, renovação de livros, consulta ao acervo, etc.

De forma geral, ambos os sites se mostraram bem qualificados quanto à questão de usabilidade, necessitando apenas de alguns ajustes que podem ser realizados com a criação de novas funções e o desenvolvimento de documentos.

\section{REFERÊNCIAS}

AGARWAL, R.; VENKATESH, V. Assessing a firm's web presence: a heuristic evaluation procedure for the measurement of usability. Information Systems Research, v. 13, n. 2, p. 168-186, 2002.

ANDRADE, António Luis Lordelo. Usabilidade de interfaces Web: Avaliação heurística no jornalismo on-line. Rio de Janeiro: E-papers, 2007.

ASSOCIAÇÃO BRASILEIRA DE NORMAS TÉCNICAS. NBR 9241-11: Informação e documentação: Requisitos Ergonômicos para Trabalho de Escritórios com Computadores Parte 11 - Orientações sobre Usabilidade: apresentação. Rio de Janeiro, 2002.

BARBOSA, S.D.J.; SILVA, B.S. Interação Humano-Computador. Rio de Janeiro: Elsevier, 2010.

BONIFÁCIO, B. A. et al. Usabilidade de Aplicações Web Móvel: Avaliando uma Nova Abordagem de Inspeção através de Estudos Experimentais. In: IBERO-AMERICAN CONFERENCE ON SOFTWARE ENGINEERING, 21.,2012, Buenos Aires. Anais...Buenos Aires: ClbSE, 2012.

CYBIS, Walter; BETIOL, Adriana Holtz; FAUST, Richard. Ergonomia e Usabilidade: conhecimentos, métodos e aplicações. 2. ed. São Paulo: Novatec Editora, 2010.

CRUZ, Allan Kássio Beckman Soares da; SOARES NETO, Carlos de Salles. Revisitando as heurísticas de avaliação de Nielsen para análise de usabilidade em jogos de tabuleiro não virtuais. Human Factors in Design, v. 3, n. 06, p. 35-47, 2015.

DIAS, C. Usabilidade na Web: criando portais mais acessiveis. Rio de Janeiro: Alta Books, 2003. 
EIGHMEY, J.; McCORD, L. Adding value in the information age: uses and gratifications of sites on the World Wide Web. Journal of Business Research, v. 41, p. 187-194, 1998.

HERRERA-VIEDMA, E. et al. Evaluating the information quality of web sites: a methodology based on fuzzy computing with words. Journal of the American Society for Information Science and Technology, v. 57, n. 4, p. 538-549, 2006.

LECEROF, A.; PATERNÒ, F. Automatic support for usability evaluation. IEEE Transactions on Software Engineering, v. 24, n. 10, p. 863-888, 1998.

LEITE, Jair Cavalcanti..Modelos e Formalismos para a Engenharia Semiótica de Interfaces de Usuário. 1998. Tese (Doutorado em Ciências da Informação) - Pontifícia Universidade Católica do Rio de Janeiro, Rio de Janeiro, 1998.

NIELSEN, Jakob; LORANGER, Hoa. Usabilidade na Web: Projetando Websites com Qualidade. Rio de Janeiro: Campus. 2007.

PORTER, Michael E. Strategy and the Internet. Massachusetts: Harvard Business Review, 2001.

SANTA ROSA, José Guilherme; MORAES, Ana Maria de: Avaliação e projeto no design de interfaces. Rio de Janeiro: $2 A B, 2008$.

SHITKOVA, M. et al. Towards Usability Guidelines for Mobile Websites and Applications. In PROCEEDINGS OF THE, 12., 2015, Osnabrück, Germany. Anais... Osnabrück: Internationalen Tagung Wirtschaftsinformatik, 2015. p. 1603-1617.

SHNEIDERMAN, Ben. Designing the User Interface - Strategies for Effective Human-Computer Interaction. Boston: Ed. Addison-Wesley, 1998. 